\section{Sublinguale Applikation im Vorteil}

D ie gute Wirksamkeit beider Darreichungsformen der systemischen Immuntherapie (SIT), subkutan als SCIT oder sublingual als SLIT, sei klar belegt, bestätigte Prof. Dr. Randolf Brehler, Münster. Das bessere Sicherheitsprofil habe dabei die SLIT. Deren Wirkung setze bereits nach vier Wochen ein, eine Effektsteigerung durch Behandlung über mehrere Jahre und ein Langzeiteffekt seien durch Studien abgesichert.
Wie Brehler ausführte, reduzierte die 5-Gräser-Tablette Oralair ${ }^{\circledR}$ bei Patienten mit allergischer Rhinitis die Symptome gegenüber Plazebo in Studien über mehrere Pollenflugperioden um bis zu 50\% im dritten Therapiejahr. Unter Pollenkammer-Bedingungen waren die Symptome bereits vier Wochen nach Therapiebeginn signifikant verbessert.

Ein evidenzbasiertes SIT-Studienprogramm zielt zudem auf verlässliche Dosisdaten ab. Brehler referierte die Er-

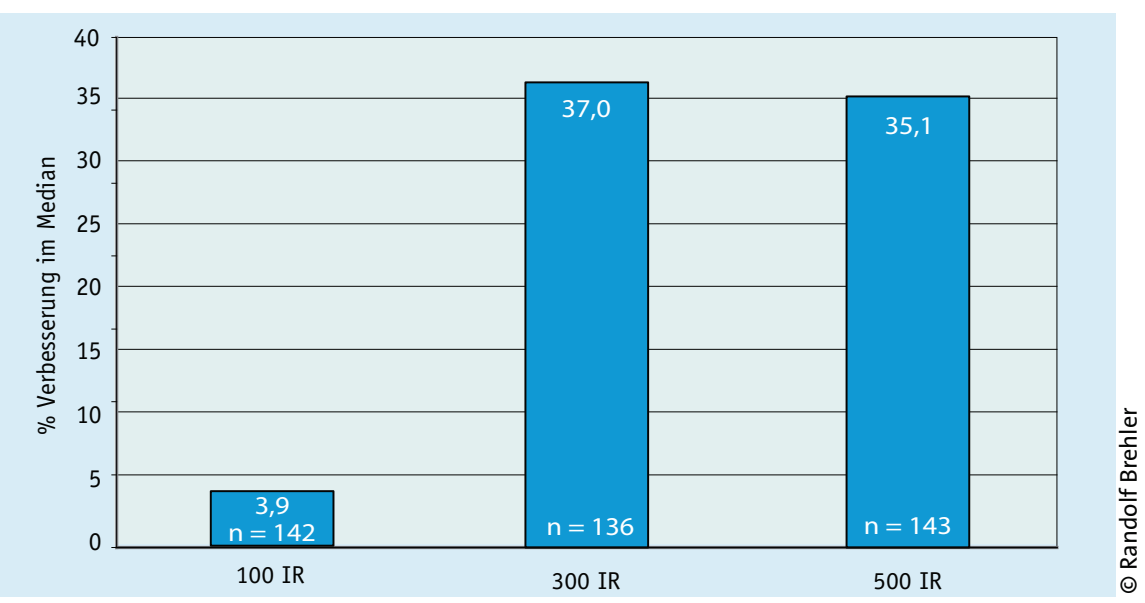

Optimaler Therapieeffekt: Eine Dosissteigerung über 300 IR hinaus führte bei der 5-Gräser-Tablette zu keiner weiteren Wirkungssteigerung.

\title{
Gerüstet für gestiegene Anforderungen
}

E. ine wirksame spezifische Immuntherapie (SIT) muss vor allem die Beschwerden der behandelten Patienten tatsächlich bessern. Wie Prof. Dr. KarlChristian Bergmann, Berlin, erläuterte, lässt sich dieser Umstand mithilfe des kombinierten Symptom-MedikationsScores (SMS) besonders gut beurteilen. Nach Malling liegt eine klinisch relevante Wirksamkeit vor, wenn der SMS in der aktiv behandelten Gruppe um mehr als 30\% über dem der Plazebogruppe liegt. Gemäß der World Allergy Organization (WAO) müssen Symptom- oder Medikationsscore oder der kombinierte SMS um mindestens 20\% und signifikant gegenüber der Plazebogruppe verbessert sein.
Doppelblinde, plazebokontrollierte Studien werden durch den nicht vorhersehbaren Pollenflug erschwert. Standardisierte Konditionen für Pollenflug, Luft- und Raumbedingungen sind nur in einer Pollenkammer gewährleistet. Wie Prof. Dr. Norbert Krug vom Institut für Toxikologie und Experimentelle Medizin (ITEM) in Hannover bestätigte, sind Pollenkammerstudien eine anerkannte Methode, die Zeit bis zum Wirkeintritt eines Therapieallergens zu ermitteln. Mit einer solchen Studie am ITEM konnte Novartis den raschen Wirkeintritt der Kurzzeitimmmuntherapie mit Depiquick ${ }^{\circledR}$-Gräser-Mix bei Patienten mit allergischer Rhinitis nachweisen: Bereits acht Wochen nach The- gebnisse der Dosisfindungsstudie zur 5-Gräser-Tablette ( $\mathrm{J}$ Allergy Clin Immunol 2007; 120: 1338-47), denen zufolge eine Dosis von 500 IR gegenüber 300 IR keinen zusätzlichen Benefit brachte. Solche Studien forderte Brehler für jedes einzelne SIT-Präparat, denn: „Therapie mit der optimalen Dosis führt zu optimalem Therapieffekt."

Die hohe Abbruchrate nach der ersten Verordnung einer SIT beschäftigte Prof. Dr. Ralph Mösges, Köln. Nach seinen Feststellungen führt weniger als die Hälfte der Patienten eine SIT konsequent durch, die Mehrheit ist vom Ergebnis der Hyposensibilisierung enttäuscht. Mösges umriss die Situation und ihre Konsequenzen: „Wir vergessen die Patienten, die nicht mehr in die Praxis kommen." Die SLIT mit ihrer besseren Drei-Jahres-Persistenz biete nun Chancen, die Adhärenz der Patienten positiv zu beeinflussen. Die Vorzüge der 5-Gräser-Tablette sah der Referent in ,geringerer zeitlicher Beanspruchung und weniger Nebenwirkungen". Jürgen Setton

Mittagssymposium „Die neue Wirklichkeit in der spezifischen Immuntherapie” im Rahmen des 46. Kongresses der Deutschen Dermatologischen Gesellschaft. Dresden, 1. April 2011. Veranstalter: Stallergenes, Kamp-Lintfort

rapiebeginn war die Symptomatik um 35\% im nasalen Symptomscore reduziert, in einer zweiten Studie hatte sie sich nach elf Wochen um $44 \%$ gebessert.

Gemäß der im November 2008 inkraftgetretenen Therapieallergene-Verordnung müssen alle wichtigen Therapieallergene zur Zulassung gebracht und deren Wirksamkeit mit doppelblinden, plazebokontrollierten Studien nachgewiesen werden. Diesen gestiegenen Anforderungen stellt sich Novartis zusammen mit seinem spanischen Partner Leti in einem umfangreichen Studienprogramm

Jürgen Setton

Journalisten-Roundtable „Klinische Studien im Wandel" im Rahmen der 46. Tagung der Deutschen Dermatologischen Gesellschaft. Dresden, 30. März 2011. Veranstalter: Novartis Pharma, Nürnberg 